\title{
NOTES ON COSMOLOGY
}

\author{
J. PACHNER \\ University of Saskatchewan, Regina, Canada
}

The present short notes on cosmology start with two conclusions based on observational data. The first of them is the well-known conclusion that the Universe from the global point of view is at the present epoch of its evolution in a uniform and isotropic expansion. If we accept the very convincing arguments of Bondi (1962) that the geometry of the cosmic space is Riemannian, its properties are described at the present epoch of the cosmic evolution by the well-known Robertson-Walker metric expressing the cosmological principle (Robertson, 1935, 1936; Walker, 1936). The form of the geodesical lines depends on the assumed theory of gravitation. For instance, in the closed Friedman world model they are cycloids with a singularity at the start of the expansion.

The 'particles of cosmic dust' that follow the cosmic expansion are generally accepted to be field galaxies or clusters of galaxies, but in cosmology let us call them 'vacuoles'. The concept of a vacuole, i.e. of a spherical region in the Friedman universe inside which all matter is concentrated to its centre, was introduced into relativistic cosmology by Einstein and Straus $(1945,1946)$. We now generalize the concept of a vacuole, defining it as a region (not necessarily of spherical shape) inside which the stars and interstellar matter move without participating in the general cosmic expansion. The dimensions and shape of these vacuoles will depend on the spatial distribution of matter inside them, and on the assumed theory of gravitation, but their dimensions, as in the case of the Einstein vacuoles, will decrease with increasing mass density in the Universe. From a certain moment of the cosmic contraction, or at the very beginning of the expansion, larger and larger parts of the outer regions of the vacuoles begin to participate in the general cosmic contraction or expansion respectively. The Robertson-Walker line element thus loses its validity and must be replaced by a new one taking into account the local rotational motion.

The second conclusion based on observational data was formulated by Hawking and Ellis (1968). They stated that "most of the matter (viz. in the Universe) avoided the singularities and re-expanded to give the present observed Universe".

A cosmological theory must agree with these two conclusions and, if we assume that the galaxies are of the same age as, or maybe of a greater age than, the age of the present expansion of the Universe, it should also explain the origin and the shape of galaxies, for instance the spiral structure of some of them.

Up to this point no assumption about the gravitational law and about the existence of other cosmic fields has been made. Now we must choose one of the following two ways. In order to save the validity of the Robertson-Walker metric for any stage of the cosmic evolution, we modify the Einstein general relativity by introducing some cosmic field - the cosmological $\Lambda$-term of Einstein (1917), the $C$-field of Hoyle (1948, 
1949, 1960), or of Hoyle and Narlikar (1964), the negative pressure of McCrea (1951), Pachner (1965a, b) and Rosen (1969) or maybe some other cosmic field.

This cosmic field must stop the contraction of the Universe and revert it to a new expansion before the rotational motion inside the vacuoles is disturbed by the contraction. From the mathematical point of view this is an easy way to remove the initial singularity of Friedman world models, but it is connected with the very difficult task of justifying the assumed modification from the physical point of view and proving that just this modification represents the true natural law of cosmic evolution.

The Einstein general relativity represents the most simple and physically bestestablished gravitational theory. We make it therefore a starting point of the other way of investigation taking into account the local rotational motion of earlier vacuoles. Let us emphasize that any relativistic world model that neglects this local rotational motion at the beginning of the expansion represents a potential world model, compatible with the Einstein field equations, but such a model does not describe the actual Universe during the period of its maximum contraction.

The situation is mathematically not so hopeless as it seems at first sight, for we must realize that the period when the local rotation could play a decisive role is relatively short. We may therefore treat the problem not as a global, but as a local one. In other words, it is not necessary to know the Cauchy data over the whole hypersurface $t=$ const., but merely over its limited, relatively small, part.

The analytical mathematical method elaborating this idea, on the simplifying assumption of an ideal fluid in which all the thermodynamic processes are adiabatic only and with no release of nuclear energy, is described in full detail elsewhere (Pachner, 1970).

It gives the following preliminary results:

(1) An extremely high pressure (so high that the relativistic limit of the equation of state must be applied) creates an additional attraction and diminishes simultaneously the influence of rotation in such a way that the occurrence of a singularity is inevitable.

(2) In rotating incoherent matter the occurrence of singularities depends on the initial data. In the case of rotational symmetry there exists a class of singularity-free Einstein fields, either with the shear or without it, but they are of no importance for the application in cosmology or astrophysics. In other cases the occurrence of a point singularity in rotating incoherent matter is inevitable. The occurrence of this type of singularity is due to the four initial conditions

$$
R_{\mu}^{4}-\frac{1}{2} R \delta_{\mu}^{4}=8 \pi T_{\mu}^{4} \quad(\mu=1,2,3,4)
$$

not the six Einstein field equations

$$
R_{i k}=8 \pi\left(T_{i k}-\frac{1}{2} T g_{i k}\right) \quad(i, k=1,2,3) .
$$

In fact it follows from the initial conditions that in rotating and simultaneously expanding or contracting incoherent matter the shear is always present. It seems plausible that the same will be true also if the assumption of rotational symmetry is dropped. 
There is no doubt that the rotation together with a pressure gradient can avoid the occurrence of singularities in an ideal fluid. The question is whether the conditions under which this is possible correspond to the observational data in the vacuoles of the actual Universe. If further investigation gives a positive answer, the problem of the initial singularity in relativistic cosmology will disappear and as a by-product we shall obtain an explanation of the origin and spiral structure of galaxies:

Due to statistical fluctuations matter concentrated into proto-galaxies during the preceding contracting phase of the Universe. Angular momentum was transferred to them by gravitational interaction with neighboring proto-galaxies during the last stages of the contracting phase. The rotation together with the pressure gradient enabled them to go through the maximum contraction of the Universe with a finite mass density. The shear, effective only during a relatively short period, gave the elongated clouds of rotating cosmic matter their spiral structure.

However, if the answer is negative, we shall obtain a cogent reason to modify the Einstein general relativity, for the big-bang hypothesis is rather an acknowledgement of certain limitations of our present knowledge than a scientific explanation of the origin of the Universe.

\section{References}

Bondi, H.: 1962, in 'Recent Developments in General Relativity', Infeld Festschrift, Pergamon Press, p. 47.

Einstein, A.: 1917, Sitzber. Preus. Akad. Wiss. p. 142.

Einstein, A. and Straus, E. G.: 1945, Rev. Mod. Phys. 17, 120.

Einstein, A. and Straus, E. G.: 1946, Rev. Mod. Phys. 18, 148.

Hawking, S. W. and Ellis, G. F. R.: 1968, Astrophys. J. 152, 25.

Hoyle, F.: 1948, Monthly Notices Roy. Astron. Soc. 108, 372.

Hoyle, F.: 1949, Monthly Notices Roy. Astron. Soc. 109, 365.

Hoyle, F.: 1960, Monthly Notices Roy. Astron. Soc. 120, 256.

Hoyle, F. and Narlikar, J. V.: 1964, Proc. Roy. Soc. London, Ser. A 278465.

McCrea, W. H.:1951, Proc. Roy. Soc. London Ser. A 206, 562.

Pachner, J.: 1965a, Monthly Notices Roy. Astron. Soc. 131, 173.

Pachner, J.: 1965b, Bull. Astron. Inst. Czech. 16, 321.

Pachner, J.: 1970, Gen. Rel. Grav. 1, 281.

Robertson, H. P.: 1935, Astrophys. J. 82, 284.

Robertson, H. P.: 1936, Astrophys. J. 83, 187, 257.

Rosen, N.: 1969, Int. J. Theor. Phys. $2,189$.

Walker, A. G.: 1936, Proc. London Math. Soc. $42,90$.

\section{Discussion}

Solheim: Will the model parameters of the contraction period be completely symmetrical with respect to the parameters of the expansion period as we observe today?

Pachner: If we consider the period when the vacuoles are already formed and compare the geodesic lines along which they move with the geodesic lines of the matter during the preceding contraction phase, we may expect that they differ from each other. In fact, it is plausible that a part of the kinetic energy of the uniform and isotropic contraction has been transformed to the rotational energy of matter inside the vacuoles, as a consequence of which the radius of space increases more slowly during the expansion than it has decreased during the contraction. 